\title{
Serum CCL-I8 level is a risk factor for COPD exacerbations requiring hospitalization
}

\author{
This article was published in the following Dove Press journal: \\ International journal of COPD \\ 5 January 2017 \\ Number of times this article has been viewed
}

\author{
Asli Gorek Dilektasli \\ Ezgi Demirdogen \\ Cetinoglu' \\ Esra Uzaslan' \\ Ferah Budak ${ }^{2}$ \\ Funda Coskun' \\ Ahmet Ursavas' \\ Ilker Ercan ${ }^{3}$ \\ Ercument Ege' \\ 'Department of Pulmonary Disesaes, \\ ${ }^{2}$ Department of Immunology, \\ ${ }^{3}$ Department of Biostatistics, Uludag \\ University Faculty of Medicine, \\ Bursa, Turkey
}

Introduction: Chemokine (C-C motif) ligand 18 (CCL-18) has been shown to be elevated in chronic obstructive pulmonary disease (COPD) patients. This study primarily aimed to evaluate whether the serum CCL-18 level differentiates the frequent exacerbator COPD phenotype from infrequent exacerbators. The secondary aim was to investigate whether serum CCL-18 level is a risk factor for exacerbations requiring hospitalization.

Materials and methods: Clinically stable COPD patients and participants with smoking history but normal spirometry (NSp) were recruited for the study. Modified Medical Research Council Dyspnea Scale, COPD Assessment Test, spirometry, and 6-min walking test were performed. Serum CCL-18 levels were measured with a commercial ELISA Kit.

Results: Sixty COPD patients and 20 NSp patients were recruited. Serum CCL-18 levels were higher in COPD patients than those in NSp patients (169 vs $94 \mathrm{ng} / \mathrm{mL}, P<0.0001)$. CCL-18 level was significantly correlated with the number of exacerbations $(r=0.30, P=0.026)$, although a difference in CCL-18 values between infrequent and frequent exacerbator COPD (168 vs $196 \mathrm{ng} / \mathrm{mL}$ ) subgroups did not achieve statistical significance $(P=0.09)$. Serum CCL-18 levels were significantly higher in COPD patients who had experienced at least one exacerbation during the previous 12 months. Overall, ROC analysis revealed that a serum CCL-18 level of $181.71 \mathrm{ng} / \mathrm{mL}$ could differentiate COPD patients with hospitalized exacerbations from those who were not hospitalized with a $88 \%$ sensitivity and $88.2 \%$ specificity (area under curve: 0.92 ). Serum CCL-18 level had a strong correlation with the frequency of exacerbations requiring hospitalization $(r=0.68, P<0.0001)$ and was found to be an independent risk factor for hospitalized exacerbations in the multivariable analysis.

Conclusion: CCL-18 is a promising biomarker in COPD, as it is associated with frequency of exacerbations, particularly with severe COPD exacerbations requiring hospitalization, as well as with functional parameters and symptom scores.

Keywords: COPD, frequent exacerbator, hospitalized exacerbation, PARC/(CCL-18)

\section{Introduction}

Chronic obstructive pulmonary disease (COPD) is a major cause of mortality and morbidity worldwide. It is a chronic and progressive disease often associated with periods of exacerbation that are related with reduced health-related quality of life, accelerated forced expiratory volume in $1 \mathrm{~s}\left(\mathrm{FEV}_{1}\right)$ decline, increased health resource utilization and increased mortality. ${ }^{1,2}$ Acute exacerbations of COPD requiring hospitalization are particularly important because they lead to an increased economical burden, and each has the potential for a fatal outcome. ${ }^{3}$ Current knowledge of factors associated with exacerbations requiring hospitalization is limited. ${ }^{4,5}$ It is suggested that COPD patients suffering from frequent exacerbations belong to a distinct phenotype, as demonstrated in the Evaluation of COPD Longitudinally to Identify Predictive Surrogate Endpoints (ECLIPSE) study. ${ }^{6,7}$
Correspondence: Asli Gorek Dilektasli Department of Pulmonary Medicine, Uludag University Faculty of Medicine Nilüfer I6059, Bursa, Turkey

Tel +90224295091।

Email asligorekd@gmail.com 
Currently, COPD diagnosis is based on the presence of symptoms and risk factors with a spirometric evaluation demonstrating $\mathrm{FEV}_{1}$ over forced vital capacity $(\mathrm{FVC})<0.70$. Since this approach has several limitations, a molecular biomarker would be of great use for diagnosing, staging, and phenotyping COPD and also for singling out the exacerbator phenotype. The potential value of several biomarkers in diagnosis, assessing the severity assessment, etiology, and frequency of COPD exacerbations has been examined in recent studies, and plausible candidates have been identified..$^{8-10}$

Pulmonary and activation-regulated chemokine (PARC)/ chemokine (C-C motif) ligand 18 (CCL-18) is a 7-kD protein that is mainly expressed by monocytes/macrophages and dendritic cells. It is secreted predominantly by the lungs, avoiding the possible interference of any protein derived from nonpulmonary organs such as the liver. ${ }^{11}$ In the ECLIPSE cohort, it was shown that serum CCL-18 levels were found to be elevated in patients with COPD and associated with COPD-related clinical and functional outcomes and mortality. ${ }^{12,13}$ The primary aim of this study was to evaluate whether levels of serum CCL-18 could differentiate the frequent exacerbator COPD phenotype from infrequent exacerbators, and the secondary aim was to investigate whether serum CCL-18 levels can predict exacerbations that would require hospitalization.

\section{Materials and methods Study subjects and study design}

This case-control study was conducted in the outpatient clinic at the Department of Pulmonary Diseases, Uludağ University Faculty Hospital - a tertiary reference center in the Southern Marmara Region, the most populous area of Turkey. COPD patients aged 40-75 years with a smoking history of $\geq 10$ pack-years, who met diagnostic criteria of the Global Initiative for Chronic Obstructive Lung Disease (GOLD) guidelines and were characterised by persistent airway limitation (post-bronchodilator $\mathrm{FEV}_{1} / \mathrm{FVC}<0.70$ ) were recruited. ${ }^{1}$ Clinically stable COPD patients with no episodes of exacerbation in the previous 2 months were consecutively enrolled from October 2012 to April 2013. COPD exacerbation was defined as an event in the natural course of the disease characterized by a change in the patient's baseline dyspnea, cough, and/or sputum that was beyond normal dayto-day variations and that required treatment with systemic steroids or antibiotics, and/or a visit to the emergency room or admission to hospital. ${ }^{14,15}$ Acute COPD exacerbations in the previous 12 months were assessed using both patients' recall of exacerbation events and medical records. Based on their frequency in the previous 12 months, COPD patients were classified as either frequent ( $\geq 2$ events, FE-COPD) or infrequent ( $<2$ events, IE-COPD) exacerbators. ${ }^{16}$ In a separate analysis, baseline characteristics of COPD patients with and without hospitalized exacerbation in the previous 12 months were compared. The control group consisted of apparently healthy volunteer participants with a smoking history of $\geq 10$ pack-years and normal spirometry (NSp). Exclusion criteria for both COPD and NSp were asthma, bronchiectasis, presence of tuberculosis, pneumonia or sign of any other active infection, inflammatory diseases such as connective tissue disorder, arthritis or inflammatory bowel disease, and malignancy. This study was approved by the ethics comittee of the Uludag University. All participants provided written informed consent prior to their inclusion in the study.

\section{Methods}

Participants were examined by one of the study researchers, and a detailed structured questionnaire including risk factors, smoking status, disease history including exacerbations, and comorbid conditions was completed for each patient. Severity of dyspnea was assessed by using the modified Medical Research Council (mMRC) Dyspnea Scale. ${ }^{17}$ Symptom severity was assessed with the eight-item patient-reported outcome measure, the COPD Assessment Test (CAT). ${ }^{18}$ The Turkish version of the CAT was shown to be a valid and reliable tool. ${ }^{19}$ Spirometry was performed before and after administration of short-acting $\beta_{2}$-agonist (albuterol) by using Vmax Encore System (Sensormedics, Viasys, Yorba Linda, CA, USA) in accordance with ATS/ ERS recommendations. ${ }^{20}$ Spirometric staging of COPD was based on GOLD guidelines. ${ }^{1}$ Subjects underwent the 6-min walking test according to the ATS statement. ${ }^{21}$ The body mass index, airflow obstruction, dyspnea, exercise capacity index (BODE index) is a multidimensional grading system comprising body mass index (BMI), airflow obstruction assessed by postbronchodilator $\mathrm{FEV}_{1} \%$ predicted, functional dyspnea based on mMRC scale, and the 6-min walking distance (6MWD) as a measure of functional exercise capacity. ${ }^{22}$ For computing the BODE index, the predescribed model was used: for each threshold value of $\mathrm{FEV}_{1}, 6 \mathrm{MWD}$, and mMRC scale, the patients received scores ranging from 0 (lowest) through 3 (maximum), while BMI was scored 0 or 1 (BMI $\leq 21$ and $>21$, respectively). Points for each variable were added, and the BODE index was calculated ranging from 0 to 10 points for each patient. ${ }^{22}$ Comorbidities were quantified by the Charlson Comorbidity Index (CCI). ${ }^{23}$ 


\section{Measurement of serum CCL- 18 and C-reactive protein (CRP) measurement}

Approximately $10 \mathrm{~mL}$ of peripheral venous blood was collected from study participants into vacutainer EDTAcontaining tubes. Serum was obtained by centrifugation at $1,500 \times \mathrm{g}$ for $10-15 \mathrm{~min}$. Samples were stored at $-80^{\circ} \mathrm{C}$ until analysed. Levels of PARC/CCL-18 were measured with a human PARC ELISA Kit (Ray Biotech, Inc) according to the manufacturer's instructions. The detection limit of this assay was $2 \mathrm{pg} / \mathrm{mL}$. Serum CRP levels were determined by an immunonephelometric method by using the BN II device (Siemens, Germany); the detection limit was $0.5 \mathrm{mg} / \mathrm{dL}$.

\section{Statistical analysis}

Statistical analyses were performed using the IBM SPSS Statistics for Windows, Version 22.0 software program (IBM Corp., Armonk, NY, USA). Variables were investigated using visual (histograms and probability plots) and analytical methods (Kolmogorov-Smirnov/Shapiro-Wilk's test) to determine whether or not they were normally distributed. Data were presented by mean \pm standard deviation. Categorical variables were reported as proportions. A chi-square test was used to compare proportions between the two groups and a two-sample $t$-test for continuous outcome variables with normal distribution (Tables 1-3). For non-normal

Table I Characteristic features of the study groups

\begin{tabular}{|c|c|c|c|}
\hline & NSp & COPD & $P$-value \\
\hline Subjects, $n$ & 20 & 60 & \\
\hline Males, n (\%) & $10(50)$ & $56(93.3)$ & $<0.000$ I \\
\hline Age, years & $59.9 \pm 12.6$ & $63.1 \pm 8.8$ & 0.25 \\
\hline \multicolumn{4}{|l|}{ Smoking status } \\
\hline Ex-smoker & $4(20)$ & $51(85)$ & $<0.0001$ \\
\hline Current smoker & $16(80)$ & $9(15)$ & \\
\hline Smoking, pack-years & $22.3 \pm 17.2$ & $49.5 \pm 24.9$ & $<0.000$ I \\
\hline Postbronchodilator $\mathrm{FEV}_{1}$, & $84.4 \pm 21.9$ & $45.7 \pm 15.2$ & $<0.0001$ \\
\hline \multicolumn{4}{|l|}{ \%predicted } \\
\hline Postbronchodilator $\mathrm{FEV} / \mathrm{FVC}$ \% & $76.8 \pm 10.5$ & $62.1 \pm 11.4$ & $<0.0001$ \\
\hline $\mathrm{BMI}, \mathrm{kg} / \mathrm{m}^{2}$ & $27.8 \pm 5.3$ & $28.1 \pm 6.8$ & 0.87 \\
\hline $\mathrm{CCl}$ & $0.43 \pm 0.8$ & $3.4 \pm 1.5$ & $<0.0001$ \\
\hline \multicolumn{4}{|l|}{ COPD severity, GOLD } \\
\hline Mild (I) & - & $\mathrm{I}(\mathrm{I} .8)$ & \\
\hline Moderate (II) & & $26(45.6)$ & \\
\hline Severe (III) & & $20(35.1)$ & \\
\hline Very severe (IV) & & $10(17.5)$ & \\
\hline
\end{tabular}

Notes: Data are presented as mean \pm SD or numbers (\%). Statistical significance was determined by the two-sample $t$-test for normally distributed continuous variables and the Mann-Whitney $U$-test for the comparison of continuous non-normal data between the groups. A chi-square test was used to compare proportions between two groups.

Abbreviations: $n$, number of subjects; NSp, normal spirometry participants; COPD, chronic obstructive pulmonary disease; $\mathrm{FEV}_{1}$, forced expiratory flow volume in I s: FVC, forced vital capacity; BMI, body mass index; $\mathrm{CCl}$, Charlson Comorbidity Index; GOLD, Global Initiative for Chronic Obstructive Lung Disease; SD, standard deviation.
Table 2 Clinical and functional characteristics of the infrequent versus frequent exacerbator COPD patients

\begin{tabular}{|c|c|c|c|}
\hline & \multicolumn{2}{|c|}{ COPD group } & \multirow[t]{2}{*}{$P$-value } \\
\hline & $\begin{array}{l}\text { IE-COPD } \\
(n=33)\end{array}$ & $\begin{array}{l}\text { FE-COPD } \\
(n=27)\end{array}$ & \\
\hline Sex, $M / F, n$ & $29 / 4$ & $27 / 0$ & 0.06 \\
\hline Age, years & $66.0 \pm 10.0$ & $67.0 \pm 8.3$ & 0.69 \\
\hline $\mathrm{BMI}, \mathrm{kg} / \mathrm{m}^{2}$ & $29.8 \pm 5.6$ & $26.2 \pm 7.6$ & 0.06 \\
\hline Smoking, pack-years & $42.6 \pm 21.3$ & $56.6 \pm 26.7$ & 0.036 \\
\hline $\begin{array}{l}\text { Postbronchodilator } \mathrm{FEV}_{1} \text {, } \\
\text { \%predicted }\end{array}$ & $50.5 \pm 13$ & $40.5 \pm 15.6$ & 0.012 \\
\hline Postbronchodilator & $65.4 \pm 8.7$ & $58.5 \pm 13.1$ & 0.026 \\
\hline $\mathrm{FEV}_{1} / \mathrm{FVC}, \%$ & & & \\
\hline mMRC & $1.4 \pm 1.1$ & $2.9 \pm 0.8$ & $<0.0001$ \\
\hline BODE & $2.0 \pm 1.2$ & $5.4 \pm 2.5$ & $<0.000$ I \\
\hline CAT & $11.6 \pm 7.4$ & $25.4 \pm 5.7$ & $<0.000$ I \\
\hline 6MWD, m & $380 \pm 68$ & $235 \pm 114$ & $<0.0001$ \\
\hline $\mathrm{CCl}$ & $3.6 \pm 1.7$ & $3.2 \pm 1.2$ & 0.042 \\
\hline CCL-18, ng/mL & $167.9 \pm 76.5$ & $195.9 \pm 72.6$ & 0.09 \\
\hline $\mathrm{CRP}, \mathrm{mg} / \mathrm{dL}$ & $1.0 \pm 1.0$ & $1.1 \pm 1.2$ & 0.52 \\
\hline Number of exacerbations/year & $I(0-I)$ & $3(2-5)$ & $<0.0001$ \\
\hline $\begin{array}{l}\text { Patients with exacerbations } \\
\text { requiring hospitalization, } \mathrm{n}(\%)\end{array}$ & $7(28)$ & $18(72)$ & $<0.000$ I \\
\hline
\end{tabular}

Notes: Data are mean \pm standard deviation or median (IQR 25-75), as appropriate. Statistical significance was determined by the two-sample $t$-test for normally distributed continuous variables and the Mann-Whitney U-test for comparison of continuous non-normal data between groups. Chi-square test was used to compare proportions between the two groups. BMI, post-bronchodilator FEV, \%predicted, mMRC score, and the 6MWD were integrated to calculate the 10-point BODE index as described previously. ${ }^{22}$

Abbreviations: COPD, chronic obstructive pulmonary disease; IE-COPD, infrequent exacerbator chronic obstructive pulmonary disease patients; FE-COPD, frequent exacerbator chronic obstructive pulmonary disease patients; $\mathrm{M}$, males; F, females; BMI, body mass index; FEV, forced expiratory flow volume in I s; FVC, forced vital capacity; mMRC, modified Medical Research Council Dyspnea Scale; BODE index, body mass index, airflow obstruction, dyspnea, exercise capacity index; CAT, COPD Assessment Test; 6MWD, 6-min walking distance; $\mathrm{CCl}$, Charlson Comorbidity Index; CCL-18, chemokine (C-C motif) ligand I8; CRP, C-reactive protein.

data, Mann-Whitney $U$-test was performed (Tables 1-3 and Figures 1 and 2). The ability of serum CCL-18 level to discriminate COPD versus NSp and to discriminate patients with COPD exacerbations in the previous 12 months who required hospitalization from those who were not hospitalized was analyzed using receiver operating characteristics (ROC) curve analysis. Optimal cutoff maximizing sensitivity and specificity was selected. Sensitivity and specificity were reported using the optimal ROC curve value according to the Youden Index. While evaluating the area under curve (AUC), a 5\% type-1 error level was used to accept a statistically significant predictive value of the test variable (Figures 3 and 4). The possible factors identified with univariable analysis were further entered into a logistic regression analysis to determine independent predictors of hospitalized COPD exacerbations. Covariates were included in the model if the $P<0.10$. Hosmer-Lemeshow goodness-of-fit test was 
Table 3 Clinical and functional characteristics of the COPD patients with and without exacerbations requiring hospitalization

\begin{tabular}{|c|c|c|c|}
\hline & \multicolumn{2}{|c|}{ COPD group } & \multirow[t]{3}{*}{ P-value } \\
\hline & \multicolumn{2}{|c|}{$\overline{\text { Hospitalized exacerbations }}$} & \\
\hline & $\begin{array}{l}0 \\
(n=35)\end{array}$ & $\begin{array}{l}\geq I \\
(n=25)\end{array}$ & \\
\hline Sex, M/F, n & $33 / 2$ & $23 / 2$ & 0.73 \\
\hline Age, years & $64.3 \pm 9.5$ & $69.7 \pm 7.8$ & 0.026 \\
\hline $\mathrm{BMI}, \mathrm{kg} / \mathrm{m}^{2}$ & $28.3 \pm 5.8$ & $27.8 \pm 8.1$ & 0.77 \\
\hline Smoking, pack-years & $44.6 \pm 21.3$ & $56.2 \pm 28.3$ & 0.11 \\
\hline Postbronchodilator & $50.4 \pm 14.8$ & $38.8 \pm 13.3$ & 0.004 \\
\hline \multicolumn{4}{|l|}{$\mathrm{FEV}_{1}$, \%predicted } \\
\hline Postbronchodilator & $66.3 \pm 9.2$ & $56.1 \pm 11.8$ & 0.001 \\
\hline \multicolumn{4}{|l|}{$\mathrm{FEV}_{\mathrm{I}} / \mathrm{FVC}, \%$} \\
\hline $\mathrm{mMRC}$ & $\mathrm{I} .7 \pm \mathrm{I} . \mathrm{I}$ & $2.7 \pm 1.2$ & 0.002 \\
\hline BODE & $2.7 \pm 2.2$ & $4.9 \pm 2.6$ & 0.003 \\
\hline CAT & $15.4 \pm 9.5$ & $21.6 \pm 8.7$ & 0.016 \\
\hline 6MWD, m & $344 \pm 99$ & $268 \pm 129$ & 0.005 \\
\hline $\mathrm{CCl}$ & $3.4 \pm 1.7$ & $3.5 \pm 1.1$ & 0.77 \\
\hline CCL-18, ng/mL & $|36.7 \pm 4| .0$ & $239.4 \pm 71.6$ & $<0.0001$ \\
\hline $\mathrm{CRP}, \mathrm{mg} / \mathrm{dL}$ & $0.8 \pm 0.8$ & $1.2 \pm 1.3$ & 0.29 \\
\hline $\begin{array}{l}\text { Number of } \\
\text { exacerbations/year }\end{array}$ & $1.6 \pm 2.1$ & $1.2 \pm 1.4$ & 0.001 \\
\hline
\end{tabular}

Notes: Data are mean \pm standard deviation or median (IQR 25-75), as appropriate. Statistical significance was determined by the two-sample $t$-test for normally distributed continuous variables and the Mann-Whitney $U$-test for the comparison of continuous non-normal data between groups. Chi-square test was used to compare proportions between the two groups.

Abbreviations: COPD, chronic obstructive pulmonary disease; $M$, males; $F$, females; BMI, body mass index; $\mathrm{FEV}_{1}$, forced expiratory flow volume in I s; FVC, forced vital capacity; mMRC, modified Medical Research Council Dyspnea Scale; BODE index, body mass index, airflow obstruction, dyspnea, exercise capacity index; CAT, COPD Assessment Test; 6MWD, 6-min walking distance; CCl, Charlson Comorbidity Index; CCL-18, chemokine (C-C motif) ligand 18; CRP, C-reactive protein

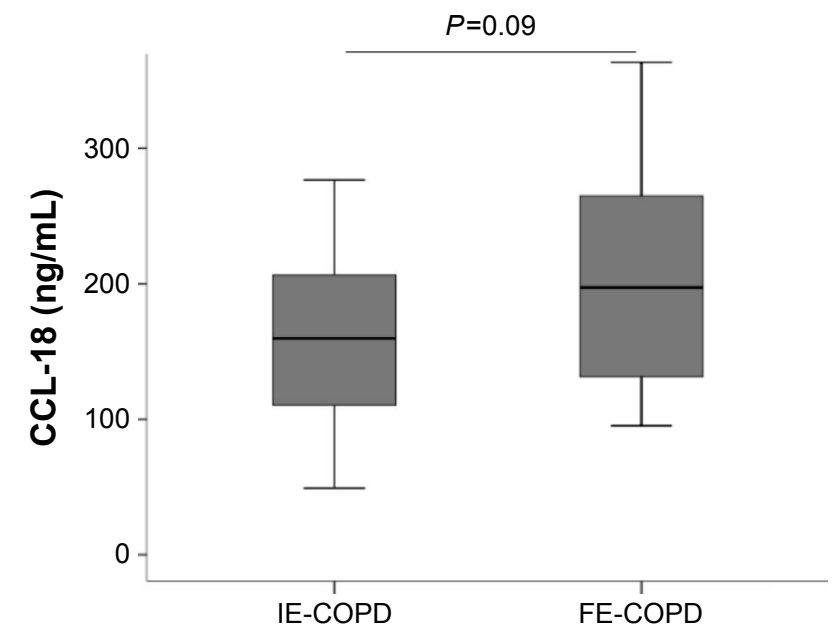

Figure I Comparison of serum CCL-I8 concentration of infrequent exacerbator COPD patients with frequent exacerbator COPD patients. The central horizontal line on each box represents the median, the ends of the boxes are 25 and 75 percentiles and the error bars $5 \%$ and $95 \%$. P-values derived from the MannWhitney U-test.

Abbreviations: COPD, chronic obstructive pulmonary disease; IE-COPD, infrequent exacerbator chronic obstructive pulmonary disease patients; FE-COPD, frequent exacerbator chronic obstructive pulmonary disease patients.

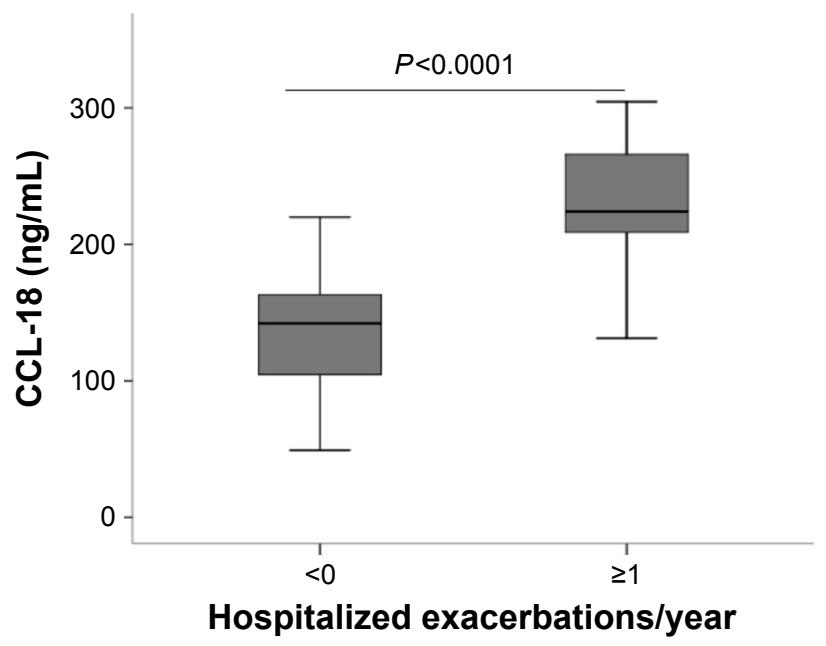

Figure 2 Comparison of serum CCL-I8 concentration in COPD patients with $\geq 1$ hospitalized exacerbations and those who were not hospitalized. The central horizontal line on each box represents the median, the ends of the boxes are 25 and 75 percentiles and the error bars $5 \%$ and $95 \%$. P-values derived from the Mann-Whitney U-test. Abbreviations: CCL-18, chemokine (C-C motif) ligand I8; COPD, chronic obstructive pulmonary disease.

used to assess model fit. Two-tailed statistical significance level was considered as 5\%, and unadjusted $P$-values were reported (Table 4). Correlations between CCL-18 and the other variables were explored using Spearman's rank correlations (Table 5 and Figure 5).

\section{Results}

\section{Subject characteristics}

Sixty COPD patients and 20 NSp patients were consecutively enrolled in the study. The characteristic and clinical features of the study subjects are outlined in Table 1. Male sex was

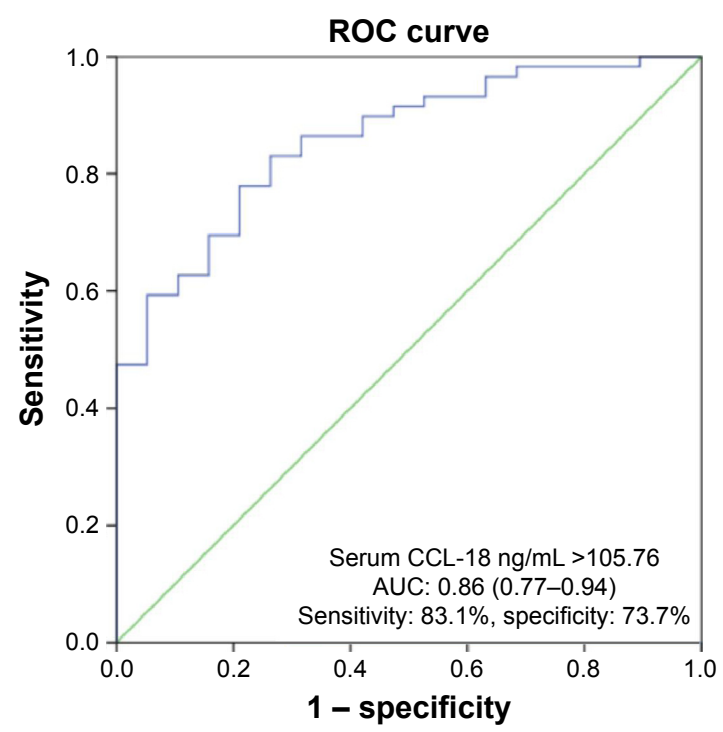

Figure 3 Receiver operator characteristic curve distinguishing COPD patients and smokers with normal spirometry AUC: 0.86 (95\% Cl: $0.77-0.94)$. The cutoff that maximizes the sum of sensitivity $(83.1 \%$ ) and specificity $(73.7 \%)$ was $>105.76 \mathrm{ng} / \mathrm{mL}$. Abbreviations: ROC, receiver operating characteristics curve anlaysis; CCL-18, chemokine (C-C motif) ligand 18; $\mathrm{AUC}$, area under curve; $\mathrm{Cl}$, confidence intervals. 


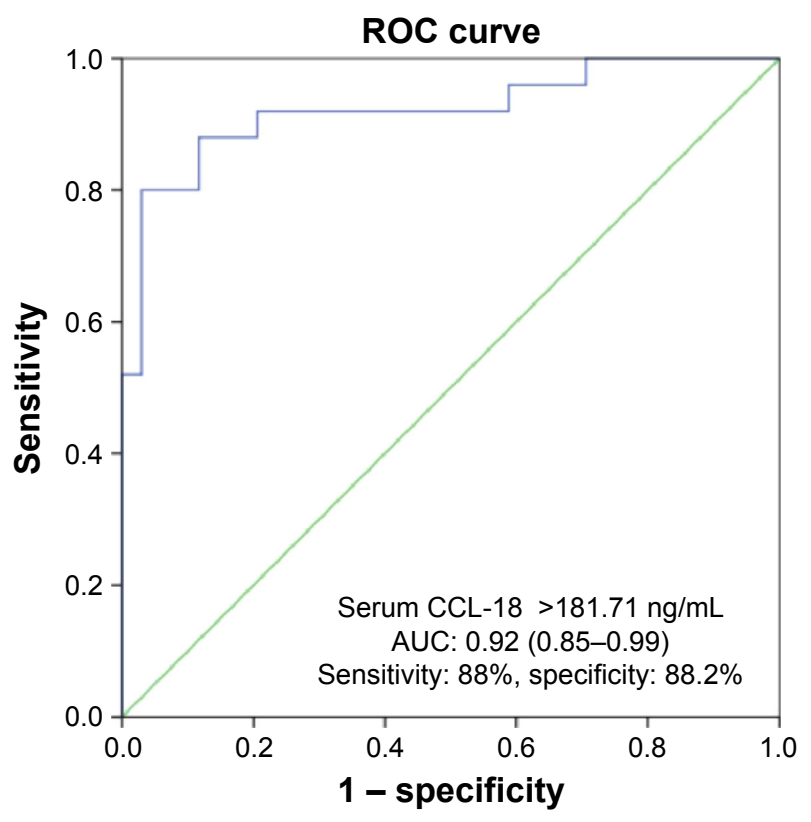

Figure 4 Receiver operator characteristic curve distinguishing COPD patients with hospitalized exacerbations from those who were not hospitalized, AUC: 0.92 (95\% Cl: $0.85-0.99)$. The cutoff that maximizes the sum of sensitivity $(88 \%)$ and specificity (88.2\%) was $>108.71 \mathrm{ng} / \mathrm{mL}$.

Abbreviations: ROC, receiver operating characteristics curve anlaysis; CCL-18, chemokine (C-C motif) ligand I8; AUC, area under curve; $\mathrm{Cl}$, confidence intervals.

more dominant among COPD patients. When the risk factors for COPD were analyzed, although only $15 \%$ of the COPD patients were current smokers, $85 \%$ were ex-smokers while $80 \%$ of the NSp group were current smokers. According to the GOLD criteria, $\sim 80 \%$ of the overall COPD patients had spirometrically moderate or severe disease (Table 1).

Of the 60 COPD patients, 27 (45\%) had frequent exacerbations and $33(55 \%)$ infrequent exacerbations in the previous 12 months. Table 2 shows the clinical characteristics of the IE-COPD and FE-COPD patients. FE-COPD patients were more likely to have higher smoking exposure, poorer $\mathrm{FEV}_{1}$ \%predicted and $\mathrm{FEV}_{1} / \mathrm{FVC} \%$ compared with IE-COPD patients. The mMRC, CAT scores, and BODE index were significantly higher; while 6MWD, and the CCI indices were lower in FE-COPD group when compared with IE-COPD patients (Table 2). FE-COPD group had significantly more exacerbations requiring hospitalization compared to IECOPD patients $(P<0.0001)$.

Of the 60 COPD patients, $25(41.7 \%)$ reported a total of 47 exacerbations requiring hospitalization in the previous 12 months prior to study admission. Of those 25, 15 (60\%) reported multiple episodes. The clinical characteristics of patients who had at least one hospitalized exacerbation versus those who were not hospitalized are summarized in Table 3. Patients with at least one hospitalized exacerbation were older. Airflow limitation, dyspnea, CAT scores, functional exercise capacity, and the BODE index were significantly worse in COPD patients who had at least one hospitalized exacerbation (Table 3 ).

\section{Serum CCL- 18 levels}

The serum CCL-18 levels were elevated in COPD patients when compared to the NSp group (180.3 \pm 75.5 vs

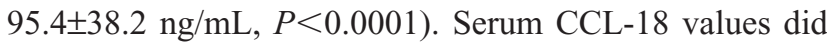
not differ significantly between the IE-COPD and FE-COPD groups, although there was a trend for FE-COPD to be higher (167.9 \pm 76.5 vs $195.9 \pm 72.6 \mathrm{ng} / \mathrm{mL}, P=0.09$ ) (Figure 1). Importantly, levels of serum CCL-18 were significantly higher in COPD patients who had at least one hospitalized exacerbation compared to those who had not been hospitalized $(P<0.0001$, Figure 2$)$. There was no difference in serum CRP levels between study groups stratified by either exacerbation phenotype or patients with a history of exacerbations requiring hospitalization (Tables 2 and 3 ).

A ROC curve analysis was carried out to evaluate the sensitivity, specificity, and the diagnostic accuracy of the serum CCL-18 levels in distinguishing COPD from NSp. The serum CCL-18 level with a threshold of $105.76 \mathrm{ng} / \mathrm{mL}$ discriminated COPD from NSp with an $83.1 \%$ sensitivity, 73.7\% specificity (AUC: $0.86 ; 95 \%$ confidence interval [CI]: $0.77-0.94$; Figure 3 ). In order to identify the optimum serum CCL-18 concentration threshold which would discriminate COPD patients with hospitalized exacerbations from those

Table 4 Univariable and multivariable analysis of possible risk factors for exacerbation requiring hospitalization in the previous year

\begin{tabular}{|c|c|c|c|c|c|c|c|c|}
\hline \multirow[t]{2}{*}{ Variables } & \multicolumn{4}{|c|}{ Univariable regression analysis } & \multicolumn{4}{|c|}{ Multivariable regression anlaysis } \\
\hline & $\mathbf{B} \pm \mathbf{S E}$ & OR & $95 \% \mathrm{Cl}$ & $P$-value & $\mathbf{B} \pm \mathbf{S E}$ & OR & $95 \% \mathrm{Cl}$ & $P$-value \\
\hline Age, years & $0.04 \pm 0.03$ & 1.04 & $0.97-1.11$ & 0.218 & - & - & - & - \\
\hline Sex & $-0.36 \pm 1.04$ & 0.70 & $0.09-5.31$ & 0.728 & - & - & - & - \\
\hline Smoking, pack-years & $0.02 \pm 0.01$ & 1.02 & $0.99-1.04$ & 0.136 & - & - & - & - \\
\hline BODE index & $0.38 \pm 0.15$ & $\mathrm{I} .46$ & $1.08-1.98$ & 0.013 & $0.44 \pm 0.29$ & 1.55 & $0.84-2.76$ & 0.133 \\
\hline CCL-18, ng/mL & $0.04 \pm 0.01$ & 1.04 & $1.02-1.06$ & $<0.000$ I & $0.05 \pm 0.02$ & 1.05 & $1.01-1.09$ & 0.014 \\
\hline Number of exacerbations & $0.35 \pm 0.16$ & 1.42 & $1.04-1.93$ & 0.026 & $0.92 \pm 0.56$ & 2.52 & $0.83-7.60$ & 0.101 \\
\hline
\end{tabular}

Note: Bold indicates significant values.

Abbreviations: BODE index, body mass index, airflow obstruction, dyspnea, exercise capacity index; SE, standard error; OR, odds ratio; Cl, confidence intervals; CCL-I8, chemokine (C-C motif) ligand 18. 
Table 5 Univariate correlations $(r)$ between serum CCL-18 level and frequency of exacerbations, frequency of exacerbations requiring hospitalization, airflow limitation, dyspnea perception, total symptom scores, 6-min walking distance, BODE index, and comorbidities in the COPD group $(n=60)$

\begin{tabular}{|c|c|c|}
\hline & \multicolumn{2}{|c|}{ CCL- $18, \mathrm{ng} / \mathrm{mL}$} \\
\hline & $r$ & $P$-value \\
\hline Smoking exposure (pack-years) & 0.45 & $<0.0001$ \\
\hline Number of exacerbations per year & 0.30 & 0.026 \\
\hline $\begin{array}{l}\text { Number of exacerbations requiring } \\
\text { hospitalization per year }\end{array}$ & 0.68 & $<0.000$ I \\
\hline Post-bronchodilator FEV, \%predicted & -0.39 & 0.001 \\
\hline Post-bronchodilator FEV/FVC, \% & -0.34 & 0.004 \\
\hline $\mathrm{mMRC}$ & 0.46 & $<0.000$ I \\
\hline CAT & $0.4 I$ & $<0.000$ I \\
\hline 6-min walking distance $(\mathrm{m})$ & -0.27 & 0.037 \\
\hline BODE & 0.58 & $<0.000 \mathrm{I}$ \\
\hline $\mathrm{SpO}_{2}$ (resting), \% & -0.45 & $<0.000$ I \\
\hline $\mathrm{CCl}^{2}$ & 0.41 & $<0.000$ I \\
\hline
\end{tabular}

Note: Spearman correlation coefficients are presented.

Abbreviations: $\mathrm{FEV}_{1}$, forced expiratory flow volume in I s; FVC, forced vital capacity; mMRC, modified Medical Research Council Dyspnea Scale; BODE index, body mass index, airflow obstruction, dyspnea, exercise capacity index; CAT, COPD Assessment Test; CCl, Charlson Comorbidity Index; CCL-I8, Chemokine (C-C motif) ligand $18 ; \mathrm{SpO}_{2}$, oxygen saturation.

who were not hospitalized, a separate ROC curve analysis was performed. The results showed that serum CCL-18 level with a threshold of $181.71 \mathrm{ng} / \mathrm{mL}$ could discriminate COPD patients with hospitalized exacerbations from those who were not hospitalized with an $88 \%$ sensitivity and $88.2 \%$ specificity (AUC: 0.92; 95\% CI: 0.85-0.99; Figure 4).

The serum CCL-18 levels were not associated with the severity of COPD based on GOLD criteria or with

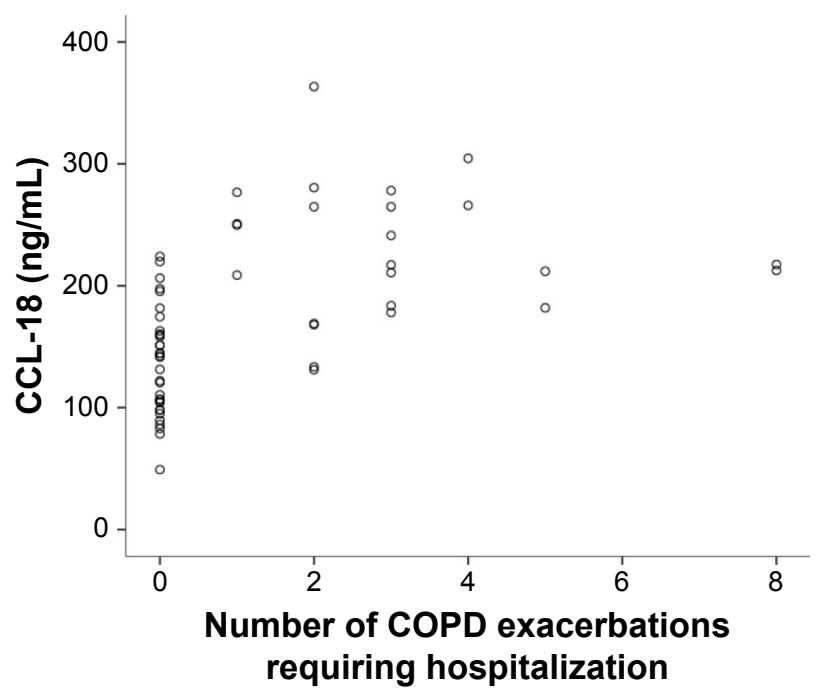

Figure 5 Scatterplot graph of serum CCL-18 concentration with number of exacerbations requiring hospitalization in COPD group.

Abbreviations: $\mathrm{CCL}-18$, chemokine (C-C motif) ligand 18; COPD, chronic obstructive pulmonary disease. the updated multidimensional COPD strategy categories $(158.0 \pm 58.6 ; 168.8 \pm 52.8 ; 164.3 \pm 65.5 ; 188.2 \pm 73.4 \mathrm{ng} / \mathrm{mL}$ for $\mathrm{A}, \mathrm{B}, \mathrm{C}$, and $\mathrm{D}$ categories respectively; $P>0.05) .{ }^{1} \mathrm{In}$ order to better understand whether smoking status was a determinant of serum CCL-18 levels, serum CCL-18 concentrations in current versus ex-smokers in both the groups were compared. In the COPD group, there was no difference in median concentrations of serum CCL-18 between current and ex-smokers $(208.0 \pm 122.2 \mathrm{vs} 175.3 \pm 64.3 \mathrm{ng} / \mathrm{mL}$, respectively, $P=0.59$ ). Although, CCL-18 levels were higher in exsmokers compared to current smokers in NSp $(117.3 \pm 47.6 \mathrm{vs}$ $89.5 \pm 34.9 \mathrm{ng} / \mathrm{mL}$ ), but the difference failed to reach statistical significance level $(P=0.18$; data not shown).

\section{Correlation of serum CCL- 18 with exacerbations, clinical and functional features of COPD}

In the COPD group, there was a significant association between the number of COPD exacerbations and CCL-18 (Spearman correlation coefficient $(r)$ of $0.30 ; P=0.026$ ). Importantly, serum CCL-18 level had a stronger correlation with the number of exacerbations requiring hospitalization $(r=0.68, P<0.0001$, Figure 5). Serum CCL-18 levels were inversely correlated with the postbronchodilator $\mathrm{FEV}_{1}$ \%predicted and postbronchodilator $\mathrm{FEV}_{1} / \mathrm{FVC}$. Elevated serum CCL-18 levels were associated with higher dyspnea perception on mMRC scale and also higher symptom scores on CAT. Decreased exercise capacity as measured by 6MWD and resting oygen saturation were inversely related to serum CCL-18 levels. There was a significant association between CCI and serum CCL-18 levels and between the serum CCL-18 levels and BODE index. Serum CCL-18 levels were positively correlated with the smoking exposure, expressed as pack-years (Table 5).

Multivariable analysis performed to define risk factors for exacerbations requiring hospitalization identified only serum CCL-18 levels (odds ratio: 1.05; 95\% CI: 1.01-1.09, $P=0.014)$ as independent predictors for hospitalized exacerbations (Table 4).

\section{Discussion}

This study sought to evaluate the serum CCL-18 levels in COPD patients subgrouped by the frequency of exacerbation, and the presence of hospitalized exacerbations in the previous 12 months. The main findings of this study are as follows: first, it was found that serum CCL-18 levels are elevated in COPD patients relative to NSp participants. Serum CCL-18 level $>105.76$ had a sensitivity of $83 \%$ and specificity of 
$74 \%$ for identifying COPD patients (AUC: 0.86). Second, it was observed that serum CCL-18 levels clearly presented significant associations with important clinical correlates of COPD such as lung functions, smoking exposure, aging, symptom scales, functional exercise capacity, and exacerbation frequency. Most importantly, serum CCL-18 levels were found to be significantly higher in COPD patients who had experienced at least one exacerbation during the previous year. ROC analysis revealed that a serum CCL-18 level of $181.71 \mathrm{ng} / \mathrm{mL}$ could differentiate COPD patients with hospitalized exacerbations in the previous 12 months from those who were not hospitalized with an $88.0 \%$ sensitivity and $88.2 \%$ specificity (AUC: 0.92 ). CCL-18 levels have strong correlations to the frequency of exacerbations requiring hospitalization, and it is an independent risk factor for hospitalized exacerbations in the multivariable analysis. Last, a trend for CCL-18 level to be higher among FE-COPD than IE-COPD patients $(P=0.09)$ was observed.

COPD is known as a disease of systemic inflammation. As the identification of biomarkers in COPD is a developing field, a topic of active research is the utility of biomarkers in improving subphenotyping of COPD patients. ${ }^{24} \mathrm{CCL}-18$ is mainly expressed by monocytes/macrophages and immature dendritic cells. It is involved in primary immune responses and induces collagen production in lung fibroblasts. ${ }^{25}$ CCL-18 was elevated in various fibrotic lung diseases such as idiopathic pulmonary fibrosis, idiopathic interstitial pneumonia, systemic sclerosis, sarcoidosis, and hypersensitivity pneumonitis. ${ }^{11,26,27}$ Similar to findings reported in two large cohorts (the Lung Health Study and ECLIPSE), ${ }^{12}$ it was observed that there was a marked increase in serum CCL-18 levels of COPD patients compared to NSp patients.

Macrophages play a key role in respiratory defense mechanisms. They have the possibility to morph into different phenotypes (M1, M2, M2-like macrophages) depending on the signals they receive from the micro-environment. M2 macrophages have poor antigen-presenting capabilities; they play a role in physiological/pathological tissue remodeling, maintenance, and progression of fibrosis; and they are considered to be wound-healing macrophages. ${ }^{28} \mathrm{CCL}-18$ was shown to be a macrophage differentiation factor and to induce an M2 spectrum macrophage phenotype..$^{29}$ There is an accumulation of M2 macrophages and, therefore, abundant collagen deposition in idiopathic pulmonary fibrosis (IPF) patients' lungs. ${ }^{30}$ The maintenance and progression of pulmonary fibrosis are postulated to be associated with M2 macrophages. ${ }^{31}$ There is also evidence supporting the role of M2 macrophage activation and dysregulation in COPD. ${ }^{28}$ Shaykhiev et al showed that M2 genes in bronchoalveolar lavage macrophages were upregulated after smoking; and this finding is more pronounced in smokers with COPD. ${ }^{32}$ Biomarker studies performed in COPD and IPF patients pointed out an overlap in elevation of several biomarkers (interleukin-6, interleukin-8, matrix metalloproteinase-1,7,9) including CCL-18 in both the diseases. ${ }^{31}$ Accordingly, it is suggested that both the diseases may share a similarity in the pathogenesis. Cigarette smoke exposure is a common and dominant risk factor for the development of both those chronic progressive parenchymal lung diseases. M2 macrophages are defective in phagocytosis and inefficient supporters of host defense mechanisms. For this reason, it is suggested that $\mathrm{M} 2$ macrophage accumulation may be responsible for repeated pulmonary infections and frequent exacerbations in both COPD and IPF patients. ${ }^{33}$

Despite a trend for CCL-18 to be higher in patients experiencing frequent exacerbations, the difference failed to achieve statistical significance in our study. In their study exploring factors associated with change in exacerbation frequency in the ECLIPSE cohort consisting of COPD patients, Donaldson et al observed higher serum CCL-18 levels in frequent exacerbators at baseline compared with the infrequent exacerbators. ${ }^{16}$ But, CCL-18 was not an imminent factor associated with changes in the exacerbation frequency categories of COPD patients. ${ }^{16}$ It seems possible that the sample size might not be large enough for a statistically significant difference in serum CCL-18 levels between IECOPD and FE-COPD groups to be observed. On the other hand, the study was successful in showing the significant correlation between serum CCL-18 levels and the frequency of the overall COPD exacerbations and exacerbations requiring hospitalization. One possible explanation for this finding might be that CCL-18 - a differentiation factor inducing M2 macrophage phenotype - may be responsible for repeated exacerbations driven by an endogenous M2-type macrophage inflammation. Importantly, serum CCL-18 levels of COPD patients who had at least one hospitalized exacerbation in the previous year were significantly higher compared to those who did not require hospitalization. This study was also able to define a threshold of serum CCL-18 $>181.71 \mathrm{ng} / \mathrm{mL}$ that differentiates COPD patients with hospitalized exacerbations from those who were not hospitalized with a high sensitivity and specificity. Whether M2-type macrophage activation is responsible for severe COPD exacerbations requiring hospitalization is unknown. It is believed that further research profiling cytokines and macrophages is required to understand the pathomechanisms for frequent exacerbations in COPD. 
The GOLD guidelines acknowledge the importance of frequent exacerbations (defined as $\geq 2$ events/year) in the disease course of COPD. FE and IE phenotypes are recognized as important COPD phenotypes that influence the clinical management of the patient. ${ }^{1}$ In the present study, frequent exacerbators were more likely to have a more severe airflow limitation consistent with the previous studies reporting an increase in exacerbation frequency as disease severity rises. ${ }^{8,34}$ Furthermore, FE-COPD patients had significantly higher CAT and mMRC scores than the IE-COPD group, also consistent with previous reports. ${ }^{35,36} \mathrm{Al}$ Ahmari et al observed a $54 \mathrm{~m}$ reduction in exercise capacity in 6MWD in FE-COPD compared to IE-COPD exacerbators. ${ }^{37}$ Interestingly, an even greater difference was observed in 6MWD between IE-COPD and FE-COPD groups ( $380 \pm 68$ vs $235 \pm 114 \mathrm{~m}$, $P<0.0001$, respectively). One explanation for this finding might be that the frequent exacerbator COPD group in this study suffered from more severe airflow obstruction. It was also found that FE-COPD patients had higher BODE indices than the infrequent exacerbators. Recently, it was shown that the BODE index - a predictor of survival in COPD - was also a good predictor of the likely number of exacerbations in COPD patients. ${ }^{38}$ Overall, the results confirm that the frequent exacerbator phenotype is a distinct subgroup in COPD with more severe clinical features and functional impairment. It is also shown that serum CCL-18 levels were correlated to postbronchodilator $\mathrm{FEV}_{1} / \mathrm{FVC}$, postbronchodilator $\mathrm{FEV}_{1}$ \%predicted, and the composite BODE index. Similarly, Pinto-Plata et al described a significant relationship between the serum CCL-18 levels and reduced $\mathrm{FEV}_{1}$ \%predicted and BODE scores. ${ }^{13}$ Despite its correlation with $\mathrm{FEV}_{1}$, in our study serum CCL-18 concentrations did not differ across the GOLD stages, in concurrence with the findings of both Sin et al and Pinto-Plata et al. ${ }^{12,13}$ Importantly, it was observed that CCL-18 had significant correlations with dyspnea, CAT symptom score, functional exercise capacity, the composite BODE index, oxygen saturation, and comorbidity impact. This study thus demonstrates a wide association between serum CCL-18 levels and hospitalized exacerbations as well as a wide range of prognostic and clinical COPD parameters.

Preliminary data support that CCL-18 expression can be modified by acute effects of smoking. ${ }^{39}$ The present study design does not enable us to evaluate the effects of acute smoking on CCL-18 in the study population; however, in the COPD group, CCL-18 levels were higher in current smokers than in ex-smokers $(P=0.59)$, whereas in the NSp group, they were higher in ex-smokers than in current smokers $(P=0.18)$, albeit with no significant difference. Investigating both the Lung Health Study and ECLIPSE cohorts, Sin et al reported that COPD patients who were ex-smokers had higher serum CCL-18 expression than current smokers. ${ }^{12}$ It is possible that the lack of association between smoking status and serum CCL-18 concentration in the present study may be a result of inadequate statistical power rather than a true lack of association. Sin et al also reported that total smoking exposure was not significantly associated with the serum CCL-18 level. Interestingly, the present study has found a positive correlation between the total smoking exposure (pack-years of smoking) and the serum CCL-18 expression $(r=0.45, P<0.0001)$. Further investigations are needed to clarify the effect of smoking on CCL-18, and whether this biomarker plays a role in the COPD pathogenesis.

There are some limitations to this study. First, this study had a cross-sectional design. The number of COPD exacerbations during the previous year was recorded based on each patient's self-report and available medical records. A prospective cohort study would enable us to evaluate the association of serum CCL-18 levels with the future exacerbations. However, it has been recently shown in the ECLIPSE cohort that hospitalized exacerbations of the past year were a strong predictor of future exacerbations requiring hospitalization. ${ }^{4}$ Second, despite CCL-18 being a predominantly pulmonary chemokine, it may be secreted by the myeloid cells, and other organs such as liver, bone marrow, and so on. Hence, measuring CCL-18 production in bronchoalveolar lavage fluid might be more revealing. However, a close correlation between CCL-18 concentration in serum and bronchoalveolar lavage was observed by Prasse et al suggesting that CCL-18 production within the lungs was reflected by the CCL-18 levels in the serum of smokers. ${ }^{11}$ Third, the prominent role of inflammatory pathways in exacerbations of COPD is well known. Although it is recognized that CRP independently contributes to COPDrelated important clinical outcomes, similar to the analysis by Müllerova et al in the ECLIPSE cohort, the present study did not observe CRP as a significant determinant for hospitalized COPD exacerbations. ${ }^{4}$ On the other hand, elevated fibrinogen levels were reported as independent risk factors for exacerbations and future hospitalized exacerbations in COPD. ${ }^{4,40}$ Since serum fibrinogen level was not analyzed, the relationship of CCL-18 with fibrinogen could not be evaluated. Last, previous research by Kollert et al showed evidence that chronic cigarette smoking alters alveolar macrophage phenotype and CCL-18 is down-regulated 
in smokers. ${ }^{39}$ In this regard, to be able to make a reliable evaluation of serum CCL-18 levels between COPD and NSp groups, only smokers and ex-smokers with at least 10 pack-years of smoking history were enrolled with NSp, as apparently healthy controls for our study. Since never smoker NSp healthy controls were not enrolled, our results regarding effect of smoking on CCL-18 are limited and should be interpreted with caution.

CCL-18 levels were elevated and associated with increased mortality in COPD patients of the ECLIPSE cohort. ${ }^{12}$ Findings in the Lung Health Study cohort indicated serum CCL-18 as an independent risk factor for higher cardiovascular hospitalization and deaths. ${ }^{12}$ In another crosssectional study profiling inflammatory phenotypes and clinical outcomes of a cohort of COPD patients, an association between elevated serum CCL-18 level and reduced FEV ${ }_{1}$, lower exercise capacity (6MWD), and frequent exacerbations were reported. ${ }^{13}$ Consistent with previous studies, it was observed that serum CCL-18 presents significant associations with all of the important clinical correlates of COPD (lung functions, smoking exposure, symptom scales, functional exercise capacity, and exacerbation frequency). Müllerova et al recently reported a comprehensive systemic inflammatory profiling (white blood cells, neutrophils, CRP, fibrinogen, and CCL-18) of the COPD patients at baseline in the ECLIPSE cohort, classified by the incidence of hospital admissions during follow-up period. Despite markers of systemic inflammation, including CCL-18 being significantly higher in COPD patients with hospitalized exacerbations compared with those without hospital admission in univariable analysis, the only inflammation markers identified as independent risk factors for future hospitalized exacerbations were serum fibrinogen and white blood cells. ${ }^{4}$ The present study reports for the first time that CCL-18 levels have a strong correlation with the frequency of hospitalized exacerbations and that serum CCL-18 level is an independent risk factor for hospitalized exacerbations.

In summary, the lung-predominant chemokine, CCL-18, could be a useful biomarker in COPD. This study demonstrates that it is significantly increased in COPD, that it has a trend to increase in COPD patients with frequent exacerbations, and that it presents a marked increase in COPD patients suffering from exacerbations requiring hospitalization. It is associated with frequency of exacerbations, exacerbations requiring hospitalization, as well as functional parameters, symptom scores, and the composite BODE index. As the validity and utility of biomarkers depend on characteristics such as association with the clinical outcomes and sensitivity in detecting clinically important differences, CCL-18 seems like a promising marker in COPD. Further studies are required to determine whether this biomarker has a causal role in the disease and has association with various COPD phenotypes.

\section{Acknowledgments}

We would like to express our sincere gratitude to Richard Casaburi, PhD, MD, and Abdullah Sayiner, MD, for their significant intellectual contribution and critically reviewing the manuscript. We also acknowledge Claire Ölmez for professional editing. Some of the results of this study have been reported in the form of an abstract (Dilektasli AG, Cetinoglu ED, Uzaslan E, Budak F, Coskun F, Ursavas A, Ege E. Feasibility of serum PARC/CCL-18 levels discriminating the frequent exacerbator COPD phenotype: a case-control study. Chest. 2014;146:61A).

\section{Disclosure}

The authors report no conflicts of interest in this work.

\section{References}

1. Vestbo J, Hurd SS, Agustí AG, et al. Global strategy for the diagnosis, management, and prevention of chronic obstructive pulmonary disease: GOLD executive summary. Am J Respir Crit Care Med. 2013; 187(4):347-365

2. Uzaslan E, Mahboub B, Beji M, et al. The burden of chronic obstructive pulmonary disease in the Middle East and North Africa: results of the BREATHE study. Respir Med. 2012;106:S45-S59.

3. Mannino DM, Buist AS. Global burden of COPD: risk factors, prevalence, and future trends. Lancet. 2007;370(9589):765-773.

4. Mullerova H, Maselli DJ, Locantore N, et al. Hospitalized exacerbations of COPD: risk factors and outcomes in the ECLIPSE cohort. Chest. 2015;147(4):999-1007.

5. Bahadori K, FitzGerald JM. Risk factors of hospitalization and readmission of patients with COPD exacerbation - systematic review. Int J Chron Obstruct Pulmon Dis. 2007;2(3):241-251.

6. Hurst JR, Vestbo J, Anzueto A, et al. Susceptibility to exacerbation in chronic obstructive pulmonary disease. N Engl J Med. 2010;363(12): 1128-1138.

7. Han MK, Agusti A, Calverley PM, et al. Chronic obstructive pulmonary disease phenotypes: the future of COPD. Am J Respir Crit Care Med. 2010;182(5):598-604.

8. Hurst JR, Donaldson GC, Perera WR, et al. Use of plasma biomarkers at exacerbation of chronic obstructive pulmonary disease. Am J Respir Crit Care Med. 2006;174(8):867-874.

9. Bozinovski S, Hutchinson A, Thompson M, et al. Serum amyloid a is a biomarker of acute exacerbations of chronic obstructive pulmonary disease. Am J Respir Crit Care Med. 2008;177(3):269-278.

10. Donaldson GC, Seemungal TA, Patel IS, et al. Airway and systemic inflammation and decline in lung function in patients with COPD. Chest. 2005;128(4):1995-2004.

11. Prasse A, Pechkovsky DV, Toews GB, et al. CCL18 as an indicator of pulmonary fibrotic activity in idiopathic interstitial pneumonias and systemic sclerosis. Arthritis Rheum. 2007;56(5):1685-1693.

12. Sin DD, Miller BE, Duvoix A, et al. Serum PARC/CCL-18 concentrations and health outcomes in chronic obstructive pulmonary disease. Am J Respir Crit Care Med. 2011;183(9):1187-1192. 
13. Pinto-Plata $V$, Casanova $C$, Müllerova $H$, et al. Inflammatory and repair serum biomarker pattern. Association to clinical outcomes in COPD. Respir Res. 2012;13(1):71.

14. Burge S, Wedzicha JA. COPD exacerbations: definitions and classifications. Eur Respir J. 2003;41:46-53.

15. Celli BR, MacNee W. Standards for the diagnosis and treatment of patients with COPD: a summary of the ATS/ERS position paper. Eur Respir J. 2004;23(6):932-946.

16. Donaldson GC, Müllerova H, Locantore N, et al. Factors associated with change in exacerbation frequency in COPD. Respir Res. 2013;14:79.

17. Bestall J, Paul E, Garrod R, Garnham R, Jones P, Wedzicha J. Usefulness of the Medical Research Council (MRC) dyspnoea scale as a measure of disability in patients with chronic obstructive pulmonary disease. Thorax. 1999;54(7):581-586.

18. Jones P, Harding G, Berry P, Wiklund I, Chen W, Leidy NK. Development and first validation of the COPD Assessment Test. Eur Respir J. 2009;34(3):648-654.

19. Yorgancıoğlu A, Polatlı M, Aydemir Ö, et al. [Reliability and validity of Turkish version of COPD assessment test]. Tuberk Toraks. 2011;60(4): 314-320. Turkish

20. Miller MR, Crapo R, Hankinson J, et al. General considerations for lung function testing. Eur Respir J. 2005;26(1):153-161.

21. ATS Committee on Proficiency Standards for Clinical Pulmonary Function Laboratories. ATS statement: guidelines for the six-minute walk test. Am J Respir Crit Care Med. 2002;166(1):111.

22. Celli BR, Cote CG, Marin JM, et al. The body-mass index, airflow obstruction, dyspnea, and exercise capacity index in chronic obstructive pulmonary disease. $N$ Engl J Med. 2004;350(10):1005-1012.

23. Charlson M, Szatrowski TP, Peterson J, Gold J. Validation of a combined comorbidity index. J Clin Med. 1994;47(11):1245-1251.

24. Nicholas BL. Search for biomarkers in chronic obstructive pulmonary disease: current status. Curr Opin Pulm Med. 2013;19(2):103-108.

25. Schutyser E, Richmond A, Van Damme J. Involvement of CC chemokine ligand 18 (CCL18) in normal and pathological processes. J Leukoc Biol. 2005;78(1):14-26.

26. Kodera M, Hasegawa M, Komura K, Yanaba K, Takehara K, Sato S. Serum pulmonary and activation-regulated chemokine/CCL18 levels in patients with systemic sclerosis: a sensitive indicator of active pulmonary fibrosis. Arthritis Rheum. 2005;52(9):2889-2896.

27. Prasse A, Pechkovsky DV, Toews GB, et al. A vicious circle of alveolar macrophages and fibroblasts perpetuates pulmonary fibrosis via CCL18. Am J Respir Crit Care Med. 2006;173(7):781-792.

28. Boorsma CE, Draijer C, Melgert BN. Macrophage heterogeneity in respiratory diseases. Mediators inflamm. 2013;2013:769214.
29. Schraufstatter IU, Zhao M, Khaldoyanidi SK, DiScipio RG. The chemokine CCL18 causes maturation of cultured monocytes to macrophages in the M2 spectrum. Immunology. 2012;135(4):287-298.

30. Stahl M, Schupp J, Jäger B, et al. Lung collagens perpetuate pulmonary fibrosis via CD204 and M2 macrophage activation. PLoS One. 2013; 8(11):e81382.

31. Doyle TJ, Pinto-Plata V, Morse D, Celli BR, Rosas IO. The expanding role of biomarkers in the assessment of smoking-related parenchymal lung diseases. Chest. 2012;142(4):1027-1034.

32. Shaykhiev R, Krause A, Salit J, et al. Smoking-dependent reprogramming of alveolar macrophage polarization: implication for pathogenesis of chronic obstructive pulmonary disease. J Immunol. 2009;183(4): 2867-2883.

33. Murray LA, Hogaboam CM. Mechanisms promoting chronic lung diseases: will targeting stromal cells cure COPD and IPF? In: Irusen EM, editor. Lung Diseases - Selected State of the Art Reviews. InTech; 2012. Available from: http://www.intechopen.com/books/lung-diseasesselected-state-of-the-art-reviews/mechanisms-promoting-chronic-lungdiseases-will-targeting-stromal-cells-cure-copd-and-ipf-. Accessed July 29, 2016.

34. Beeh KM, Glaab T, Stowasser S, et al. Characterisation of exacerbation risk and exacerbator phenotypes in the POET-COPD trial. Respir Res. 2013;14:116.

35. Mackay AJ, Donaldson GC, Patel AR, Jones PW, Hurst JR, Wedzicha JA. Usefulness of the Chronic Obstructive Pulmonary Disease Assessment Test to evaluate severity of COPD exacerbations. Am J Respir Crit Care Med. 2012;185(11):1218-1224.

36. Lee SJ, Lee SH, Kim YE, et al. Clinical features according to the frequency of acute exacerbation in COPD. Tuberc Respir Dis (Seoul). 2012;72(4):367-373.

37. Al Ahmari AD, Patel A, Kowlessar B, et al. COPD exacerbation frequency phenotype and exercise capacity. Am J Respir Crit Care Med. 2013;187:A1440.

38. Marin JM, Carrizo SJ, Casanova C, et al. Prediction of risk of COPD exacerbations by the BODE index. Respir Med. 2009;103(3): 373-378.

39. Kollert F, Probst C, Müller-Quernheim J, Zissel G, Prasse A. CCL18 production is decreased in alveolar macrophages from cigarette smokers. Inflammation. 2009;32(3):163-168.

40. Groenewegen KH, Postma DS, Hop WC, Wielders PL, Schlosser NJ, Wouters EF. Increased systemic inflammation is a risk factor for COPD exacerbations. Chest. 2008;133(2):350-357.
International Journal of COPD

\section{Publish your work in this journal}

The International Journal of COPD is an international, peer-reviewed journal of therapeutics and pharmacology focusing on concise rapid reporting of clinical studies and reviews in COPD. Special focus is given to the pathophysiological processes underlying the disease, intervention programs, patient focused education, and self management protocols.

\section{Dovepress}

This journal is indexed on PubMed Central, MedLine and CAS. The manuscript management system is completely online and includes a very quick and fair peer-review system, which is all easy to use. Visit http://www.dovepress.com/testimonials.php to read real quotes from published authors. 